\title{
ANÁLISE DA MASSA ESPECÍFICA E DA RETRATIBILIDADE DA MADEIRA DE Tectona grandis NOS SENTIDOS AXIAL E RADIAL DO TRONCO
}

\author{
Ghislaine Miranda Bonduelle ${ }^{1}$, Setsuo Iwakiri ${ }^{1 *}$, Rosilani Trianoski ${ }^{1}$, José Guilherme Prata ${ }^{1}$, \\ Vinicius Yurk da Rocha ${ }^{2}$ \\ ${ }^{1}$ Universidade Federal do Paraná, Departamento de Engenharia e Tecnologia Florestal, Curitiba, Paraná, Brasil - ghislaine@ufpr.br; \\ *setsuo@ufpr.br; rosilani@ufpr.br; jgprata@ufpr.br \\ ${ }^{2}$ Universidade Federal do Paraná, Curso de Engenharia Florestal, Curitiba, Paraná, Brasil - vinyrocha@ @otmail.com
}

Recebido para publicação: 20/05/2013 - Aceito para publicação: 29/03/2015

\begin{abstract}
Resumo
O objetivo deste trabalho foi avaliar a massa específica e a retratibilidade da madeira nos sentidos axial e radial do tronco. Foram coletadas cinco árvores com 18 anos, provenientes do município de Brasnorte, MT, as quais foram seccionadas nas posições da base, meio e topo, para avaliação das propriedades no sentido axial. Da parte diametral das toras foram obtidos pranchões onde foram controladas as posições radiais. A avaliação da massa específica e da retratibilidade da madeira seguiu as recomendações da norma panamericana. Os resultados demonstraram que a teca possui massa específica aparente a $12 \%$ de $0,606 \mathrm{~g} \cdot \mathrm{cm}^{-3}$, massa especifica básica de $0,524 \mathrm{~g} \cdot \mathrm{cm}^{-3}$ e fator anisotrópico de 2,271. Não foram observadas grandes variações de propriedades tanto no sentido axial quanto no radial. As propriedades físicas demonstram que a madeira possui média estabilidade dimensional, podendo ser indicada para aplicações em laminação, lambris, portas, decoração e movelaria.

Palavras-chave: Teca; anisotropia; estabilidade dimensional; qualidade da madeira.
\end{abstract}

\begin{abstract}
Analysis of wood density and shrinkage of Tectona grandis wood in axial and radial direction of the tree trunk. This research aimed to evaluate density and shrinkage of wood in axial and radial directions of the tree. We collected five trees aged 18, from the city of Brasnorte, MT, which were cut at the base, middle and top, for evaluation of the properties in axial direction. We obtained planks from the diametric part of the logs, from which we controlled radial positions. The evaluation of density and shrinkage of wood followed the panamerican standard recommendations. The results revealed that teak has apparent density at $12 \%$ of $0.606 \mathrm{~g} . \mathrm{cm}^{-3}$, basic density $0.524 \mathrm{~g} . \mathrm{cm}^{-3}$, and anisotropic fator of 2,271. There were no major variations in properties both in axial and radial direction. The physical properties demonstrate that the timber has medium dimensional stability, and it could be used in peeling, sidings, doors, decorating and furniture industry.

Keywords: Teak; anisotropy; dimensional stability; wood quality.
\end{abstract}

\section{INTRODUÇÃO}

A madeira de Tectona grandis L. f. (teca), por sua beleza, resistência mecânica e durabilidade é considerada uma das mais valiosas do mundo, e seu preço, muitas vezes, supera o preço de outras madeiras nobres. Segundo Motta (2011) o preço Free On Board (FOB) do metro cúbico de madeira de teca comercial varia de US\$400,00 a US\$3.000,00, dependendo da qualidade, ou seja, do diâmetro das toras e da presença ou não de nós, e pode ter valor até três vezes maior que o valor do mogno.

Esta espécie é originária da Índia, Mianmar, Laos e Tailândia, e tem sido aclimatada em Java e na Indonésia, onde provavelmente foi introduzida de 400 a 600 anos atrás. Sua distribuição compreende a região entre as latitudes $10^{\circ} \mathrm{N}$ a $23^{\circ} \mathrm{N}$ no sudeste da Ásia, porém, plantações bem sucedidas estendem-se desde os $28^{\circ} \mathrm{N}$ até os $18^{\circ} \mathrm{S}$ compreendendo o sudeste de Ásia, Austrália, África e América Latina. A região que mais concentra a área plantada é a Ásia tropical (94\%), seguida da África tropical (4,5\%) e dos países da América tropical (0,5\%) (PANDEY; BROWN, 2000). 
No Brasil, a T. grandis foi introduzida na década de 60, no município de Cáceres, MT, e segundo dados da Associação Brasileira de Produtores de Florestas Plantadas (ABRAF) (2013), os plantios ocuparam em 2012, uma área de aproximadamente 67 mil hectares e estão localizados principalmente nos estados do Mato Grosso, Pará e Roraima. As condições climáticas adequadas e favoráveis para o desenvolvimento da espécie em território brasileiro proporcionam taxas de crescimento superiores às dos plantios da maioria dos países produtores dessa madeira, obtendo madeira de dimensões comerciais em ciclos de 20 a 25 anos, os quais estimulam a implantação de plantios comerciais no país (FLÓREZ, 2012). Segundo a ABRAF (2013), nos estados inicialmente citados (MT, PA e RR), o incremento médio anual é de $15 \mathrm{~m}^{3} / \mathrm{ha} /$ ano, resultando num volume de madeira de 300 a $375 \mathrm{~m}^{3} / \mathrm{ha}$ ao final da rotação.

Embora a introdução da $T$. grandis em território brasileiro tenha ocorrido na década de 60 , escassos são os estudos relativos à espécie, principalmente em relação à qualidade da madeira (FLÓREZ, 2012), sendo esta, a soma de todas as características e propriedades que determinam o rendimento dos produtos finais e suas adequações para as aplicações pretendidas, entre as quais se destacam as propriedades anatômicas, físicas, químicas e mecânicas.

As propriedades físicas estão entre às de maior importância, e seu conhecimento é fundamental pois está associado às diversas formas de transformação industrial, bem como às variadas formas de utilização deste material em diferentes produtos. A massa específica é considerada a mais importante segundo Kollmann e Côté (1968), e normalmente é o ponto de partida no estudo da qualidade da madeira, podendo prever ou estimar o comportamento da madeira, auxiliar na otimização da produção da madeira, dos tratos silviculturais e na elaboração e a condução dos planos de corte, e ainda, ser um subsídio para avaliação econômica da floresta. As indústrias de base florestal também a consideram como uma das mais importantes já que é de fácil determinação e está correlacionada com rendimentos e características de diversos produtos.

Ainda em relação às propriedades físicas, a retratibilidade também é de elevada importância, principalmente quando se utiliza a madeira em produtos sólidos. De maneira geral, as variações dimensionais e a anisotropia são características indesejáveis, exigem técnicas adequadas de processamento e utilização, afetam diretamente a estabilidade dimensional e podem inviabilizar a geração de determinados produtos (DURLO; MARCHIORI, 1992). Seu estudo é essencial para a utilização da madeira tanto na construção civil, como na produção de móveis, bem como na geração de outros produtos de maior valor agregado, como portas, pisos, molduras, lambris, janelas, etc.

Quando se efetua a avaliação destas propriedades visando o conhecimento do comportamento da madeira e a indicação de utilização, deve-se considerar os vários fatores que influenciam os resultados, como por exemplo, espécie, teor de umidade, madeira juvenil, percentual de lenho tardio e inicial, bem como, a posição no fuste. A posição no fuste é um fator bastante relevante, pois durante o crescimento da árvore, fatores ambientais e intrínsecos à própria espécie determinam o grau de variação da massa específica no sentido radial e longitudinal, e consequentemente na retratibilidade, podendo servir como parâmetro para separar a madeira em classes de qualidade em função da posição de onde é retirada a peça (KOLLMAN; CÔTÉ, 1968).

Neste contexto, considerando que as propriedades físicas da madeira são de suma importância as mais variadas aplicações industriais e partindo da hipótese que a madeira, devido a sua heterogeneidade e anisotropia nos diferentes sentidos de crescimento do lenho resulta em peças com propriedades diferenciadas que influenciam na sua utilização, esse trabalho teve como objetivo avaliar a massa específica e a retratibilidade da madeira de $T$. grandis com 18 anos de idade cultivada em Brasnorte, MT, bem como, analisar as variações nos sentidos axial e radial do tronco.

\section{MATERIAL E MÉTODOS}

Foram coletadas cinco árvores de $T$. grandis, provenientes de plantios com idade de 18 anos, localizados no município de Brasnorte, estado do Mato Grosso, a uma altitude de 317 m, latitude $12^{\circ} 09^{\prime} 18^{\prime \prime}$ Sul e longitude 5758'44" Oeste. As árvores, com Diâmetro na Altura do Peito (DAP) entre 25 a $35 \mathrm{~cm}$ e altura de 10 a $12 \mathrm{~m}$, foram seccionadas obtendo-se três toras de $3 \mathrm{~m}$ cada, retiradas da base, meio e topo. Posteriormente, obtiveram-se pranchões de $7,5 \mathrm{~cm}(75 \mathrm{~mm})$ de espessura da parte diametral da tora, a qual variava de $35,0 \mathrm{~cm}$ a $15,0 \mathrm{~cm}(350 \mathrm{~mm}$ a $150 \mathrm{~mm})$, ao longo do fuste. Das extremidades dos pranchões foram retiradas peças com $35,0 \mathrm{~cm}(350 \mathrm{~mm})$ de comprimento, os quais resultaram em corpos-de-prova para determinar a massa específica aparente e básica, retratibilidade e fator anisotrópico. Na posição radial avaliou-se a madeira próxima a medula, posição intermediária e próxima a casca. A figura 1 apresenta o procedimento de amostragem. 

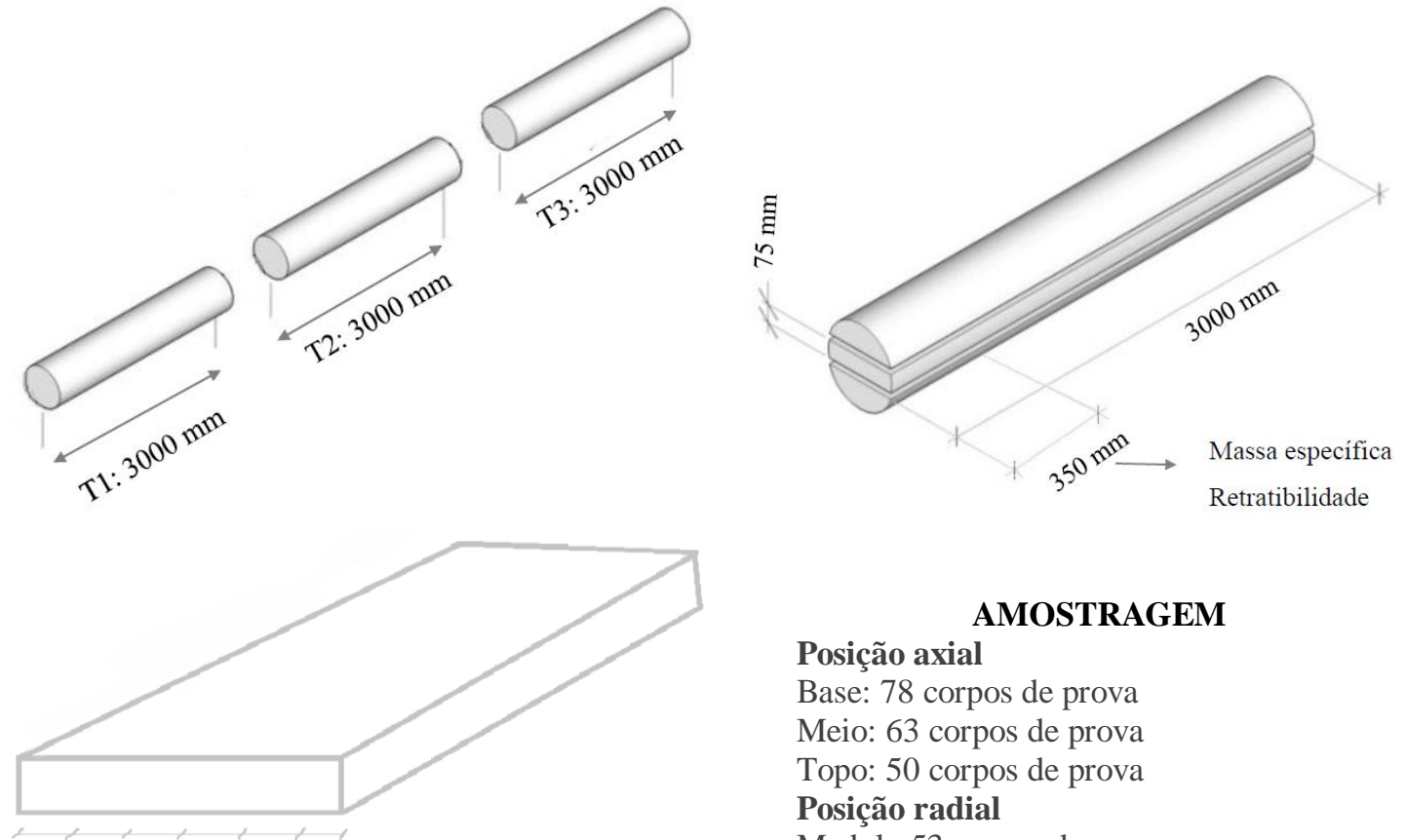

$\begin{array}{llllll}C & \text { PI } & \mathrm{M} & \mathrm{M} & \mathrm{PI} & \mathrm{C}\end{array}$

M: Medula

PI: Posição intermediária

C: Casca

AMOSTRAGEM
Posição axial
Base: 78 corpos de prova
Meio: 63 corpos de prova
Topo: 50 corpos de prova
Posição radial
Medula:53 corpos de prova
Posição intermediária: 60 corpos de prova
Casca: 55 corpos de prova

Figura 1. Esquema de retirada e amostragem dos corpos de prova de Tectona grandis.

Figure 1. Layout and sampling of Tectona grandis samples.

As peças de $350 \mathrm{~mm}$ de todas as toras ao longo do fuste, foram inicialmente, desdobradas ao meio gerando duas peças menores, e em seguida foram seccionadas segregando-se pequenos blocos da região da medula, parte intermediária e casca. A partir de cada bloco foram confeccionados o maior número possível de corpos de prova, isentos de defeitos, cujas dimensões finais nominais foram $25 \mathrm{x} 25 \mathrm{x}$ $100 \mathrm{~mm}$ (espessura, largura e comprimento, respectivamente).

Após a confecção dos corpos de prova, os mesmos foram medidos e pesados em seu estado saturado, com umidade superior a 30\%, com $12 \%$ após climatização e a $0 \%$ de umidade, de acordo com os procedimentos recomendados pela norma panamericana (COPANT 459:1972, 461:1972 e 462:1972), para determinação da massa específica e da retratibilidade.

Os resultados obtidos foram submetidos à análise estatística para avaliar diferenças entre as diferentes posições radiais e longitudinais, sendo efetuados os testes Bartlett para avaliação da homogeneidade de variância e Análise de Variância. Quando rejeitada a hipótese de igualdade, foi efetuado o teste de comparação múltipla de médias de Tukey. Foram também realizadas análises de regressão e correlações de Pearson. Todos os testes foram efetuados no Programa Statgraphics Centurion $X V$, a $95 \%$ de probabilidade.

\section{RESULTADOS E DISCUSSÕES}

\section{Valores médios de massa específica e contração da madeira}

Os valores médios de massa específica aparente e básica, contrações volumétrica, tangencial, radial e longitudinal, e fator anisotrópico, estão apresentados na tabela 1.

Verifica-se na tabela 1 que as árvores coletadas e estudadas são classificadas como de massa especifica média, segundo Melo et al. (1990), que mencionam que esta classe apresenta valores no intervalo de $0,510 \mathrm{~g} \cdot \mathrm{cm}^{-3}$ a $0,720 \mathrm{~g} . \mathrm{cm}^{-3}$. Além disso, obteve-se coeficientes de variação relativamente 
baixos, e de acordo com o intervalo apresentado pelo Forest Products Laboratory (2010), que descreve que a magnitude de variação até $10 \%$ para esta propriedade pode ser assumida. Os baixos coeficientes de variação encontrados indicam que o material apresenta-se relativamente homogêneo nas diferentes partes do fuste, o que torna-se um fator importante, visto que as demais propriedades da madeira, principalmente as mecânicas, tem uma forte correlação com a massa específica e em função disso, também não devem apresentar uma variação acentuada.

Tabela 1. Valores médios de massas específicas, contrações e fator anisotrópico da madeira de Tectona grandis.

Table 1. Average values of densities, contractions and anisotropic fator of Tectona grandis wood.

\begin{tabular}{lcccccc}
\hline $\begin{array}{l}\mathbf{M E}_{\text {aparente 12\% }} \\
\mathbf{g . c m}^{-3}\end{array}$ & $\begin{array}{c}\mathbf{M E}_{\text {básica }} \\
\mathbf{g . c m}\end{array}$ & $\begin{array}{c}\boldsymbol{B}_{\text {volumétrica }} \\
\mathbf{\%}\end{array}$ & $\begin{array}{c}\boldsymbol{B}_{\text {tangencial }} \\
\mathbf{\%}\end{array}$ & $\begin{array}{c}\boldsymbol{B}_{\text {radial }} \\
\mathbf{\%}\end{array}$ & $\begin{array}{c}\boldsymbol{B}_{\text {longitudinal }} \\
\mathbf{\%}\end{array}$ & Ac \\
\hline 0,606 & 0,524 & 6,309 & 4,205 & 1,877 & 0,343 & 2,271 \\
$(9,45)$ & $(9,94)$ & $(20,32)$ & $(21,67)$ & $(23,46)$ & $(73,46)$ & $(16,44)$ \\
\hline
\end{tabular}

MEa12\%: massa específica aparente a 12\% de umidade; MEb: massa específica básica; $\beta$ : Contração $\mathrm{A}_{\mathrm{c}}$ : Fator anisotrópico;

Valores entre parênteses referem-se ao coeficiente de variação.

Ainda, os resultados médios obtidos para a massa específica aparente e básica, de $0,606 \mathrm{~g}_{\mathrm{cm}} \mathrm{cm}^{-3} \mathrm{e}$ $0,524 \mathrm{~g} . \mathrm{cm}^{-3}$, respectivamente, apresentam-se similares aos encontrados por Mota (2011) que obteve o valor médio de $0,620 \mathrm{~g} . \mathrm{cm}^{-3}$ para massa específica aparente a $15 \%$ e $0,540 \mathrm{~g} . \mathrm{cm}^{-3}$ para massa específica básica, a partir da madeira de T. grandis com 15 anos de idade cultivada no estado de Minas Gerais; aos de Castro e Raigosa (2000), cujo valor médio foi de $0,580 \mathrm{~g} . \mathrm{cm}^{-3}$ para massa específica básica de árvores com 17 anos cultivadas na Costa Rica; aos de Gutiérrez et al. (2008) que relataram valores de 0,480 a $0,520 \mathrm{~g} . \mathrm{cm}^{-3}\left(\mathrm{ME}_{\text {básica }}\right)$ em plantios no Equador; e, aos descritos por Tsoumis (1991), que apresentou valores de massa específica entre 12 e $15 \%$ de umidade de $0,670 \mathrm{~g} \cdot \mathrm{cm}^{-3}$. Ressalta-se ainda, que as pequenas diferenças encontradas entre as pesquisas estão relacionadas à inúmeros fatores, como por exemplo procedência, sítio de crescimento, idade, metodologia de ensaio, etc.

Outro fator bastante interessante a ser mencionado diz respeito ao ciclo de produção desta espécie, que, na região de origem é de aproximadamente 80 anos. De acordo com alguns estudos tecnológicos na região de ocorrência natural, a madeira de $T$. grandis oriunda de florestas plantadas com idade entre 50 a 80 anos no Timor Leste apresentou massa específica de $0,600 \mathrm{~g} . \mathrm{cm}^{-3}$ (MIRANDA et al., 2011), 0,620 g. $\mathrm{cm}^{-3} \mathrm{em}$ árvores procedentes da Tailândia, $0,690 \mathrm{~g} \cdot \mathrm{cm}^{-3} \mathrm{em}$ material da Indonésia e $0,700 \mathrm{~g} \cdot \mathrm{cm}^{-3}$ a partir de árvores de Mianmar (BAILLÉRES; DURAND, 2000). Com base nestas informações, verifica-se que a redução do ciclo de produção de madeira de 50-80 anos na região de origem para aproximadamente 25 anos no Brasil não afetou acentuadamente a massa específica, mantendo, de certa forma propriedades similares, no entanto, havendo um elevado ganho em termos de tempo de rotação ou produção de madeira, corroborando a sua excelente adaptação e crescimento em território brasileiro.

Em relação aos valores médios das contrações e da anisotropia encontrados, a contração tangencial relativamente baixa $(4,205 \%)$ e anisotropia de contração média - alta $(2,271 \%)$, sugere que esta madeira pode ter estabilidade dimensional média a boa, não estando sujeita a empenamentos acentuados com a variação da umidade de equilíbrio das peças. Deve-se ressaltar que o fator anisotrópico tomado de forma isolada não caracteriza a madeira do ponto de vista da estabilidade dimensional e reforçar que madeiras com fator anisotrópico alto e fator de contração baixo podem ser estáveis dimensionalmente. A informação de uma boa estabilidade dimensional pode ser corroborada segundo o Forest Products Laboratory (2010), que relata que a espécie é reconhecida por uma boa estabilidade dimensional e boa resistência a água, as quais são conferidas em grande parte pelas ceras e óleos presentes nos extrativos.

Observa-se ainda, que os valores mensurados apresentaram-se próximos aos descritos por Tsoumis (1991), de 4,4\% a 9,1\% para contração tangencial, 2,1\% a 5,1\% para contração radial, e de 6,8\% a 14,0\% para contração volumétrica. Considerando os coeficientes de variação, verifica-se que os valores apresentaram-se ligeiramente superiores aos sugeridos pelos Forest Products Laboratory (2010), de 14\%, $15 \%$ e $16 \%$ para contração tangencial, radial e volumétrica, respectivamente, indicando maior variabilidade de propriedades. Já a variação apresentada pela contração longitudinal foi bastante elevada, a qual, normalmente é justificada pelo equipamento utilizado na coleta das dimensões. 
Em comparação com pesquisas disponíveis na literatura, nota-se que Mota (2011) obteve valores médios para a contração tangencial de $4,09 \%$, radial de $2,06 \%$, longitudinal de $0,29 \%$ e volumétrica de $6,33 \%$ a partir de árvores proveniente de Minas Gerais; Flórez (2012) encontrou resultados médios de $4,21 \%, 2,40 \%, 6,84 \%$ para contrações tangencial, radial e volumétrica, respectivamente, e anisotropia de contração de 1,82; Castro e Raigosa (2000) encontraram para teca plantada na Costa Rica, contrações tangencial, radial e volumétrica de 3,90\%,2,20\% e 6,20\%, respectivamente; Gutiérrez et al. (2008) relatam contrações tangenciais entre 5,22\% a 5,66\% para plantios no Equador e Tsoumis (1991) relatou valores de $5,8 \%$ para a contração tangencial, 3,0\% para contração radial e $0,6 \%$ para contração longitudinal.

Em comparação com informações de retratibilidade de árvores com crescimento na região de origem, e em especial com elevados ciclos de rotação (50-80 anos), verifica-se que Miranda et al. (2011), obtiveram valores de $5,17 \%, 3,6 \%$ e $7,6 \%$ para as contrações tangencial, radial e volumétrica, respectivamente, e consequentemente uma anisotropia de contração de 1,45\%. Tais valores apresentamse, em termos de estabilidade dimensional, superiores ao deste estudo, o qual é justificado pela formação de madeira adulta, a qual é reconhecidamente mais estável.

\section{Variações da massa específica e da retratibilidade da madeira nos sentidos axial e radial} Variação no sentido axial

A figura 2 mostra uma tendência de redução das massas específicas básica e aparente (12\%) no sentido da base ao meio do tronco, com um ligeiro aumento do meio ao topo. Entretanto, esses resultados quando analisados a um nível de significância de 5\%, demonstram que estatisticamente não existem diferenças significativas entre as duas posições mais elevadas das árvores avaliadas.

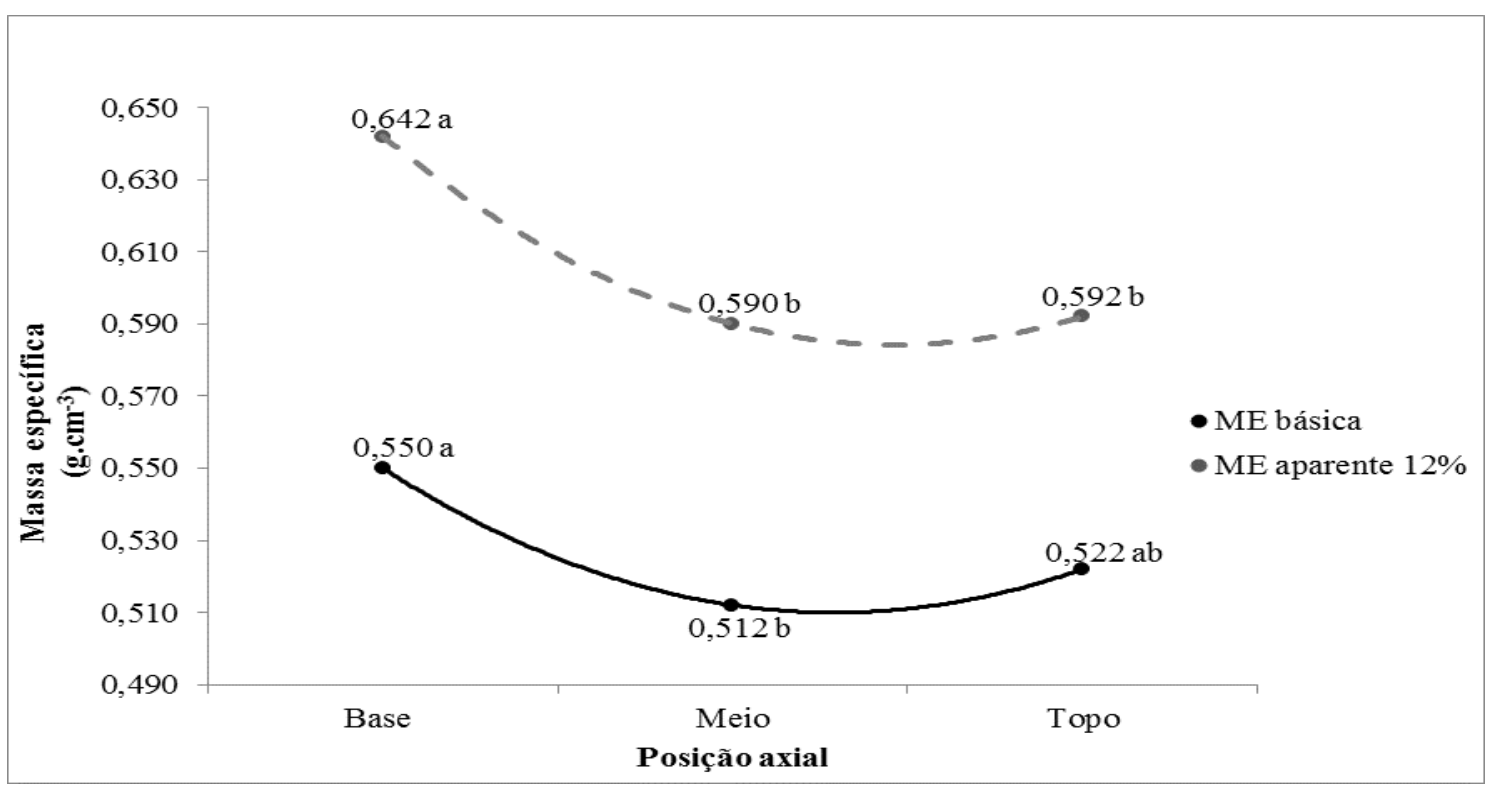

Figura 2. Variação da massa específica básica e aparente a $12 \%$ no sentido axial do tronco de Tectona grandis.

Figure 2. Variation of basic and apparent (12\%) density in the axial direction of tree trunk of Tectona grandis.

Resultado similar foi encontrado por Arruda et al. (2012) que verificaram pequeno decréscimo da massa específica básica em $T$. grandis até $25 \%$ da altura comercial e um progressivo aumento a partir de 50\% da altura. Flórez (2012) também observou a redução da massa especifica até aproximadamente $70 \%$ da altura do fuste, um ligeiro aumento nesta região, com posterior redução até o topo e, Roque e Ledezma (2003) ao avaliarem árvores de 10 anos também encontraram uma diminuição da densidade básica na parte média do fuste. Este comportamento apresentado pela madeira de $T$. grandis também foi observado em estudos conduzidos por outros pesquisadores com outras espécies, como Lopes et al. (2011) em E. grandis, E. urophylla e E. dunnii. 
Segundo Panshin e De Zeeuw (1980) não existe um padrão definido que explique o comportamento da densidade no sentido longitudinal e, embora o decréscimo ao longo do fuste seja o mais comum, existem modelos de variação em que a densidade decresce na parte inferior do tronco, seguida de um acréscimo regular nos níveis superiores da árvore; e, acréscimo em direção ao topo, porém desuniforme. Com base nos resultados obtidos, e também na classificação proposta por Melo et al. (1990), é possível afirmar que, as toras localizadas próximo à base apresentam uma melhor qualidade, no entanto, a segregação das toras por altura torna-se onerosa e possivelmente sem efeitos num processo industrial pela pequena variação da propriedade entre posições.

$\mathrm{Na}$ tabela 2 são apresentados os valores médios das contrações tangencial, radial, longitudinal e volumétrica, assim como o fator anisotrópico, nas diferentes posições longitudinais do fuste de $T$. grandis.

Tabela 2. Valores médios da retratibilidade no sentido axial do tronco de Tectona grandis.

Table 2. Average values of shrinkage in the axial direction of tree trunk of Tectona grandis.

\begin{tabular}{lccccc}
\hline Posição & $\begin{array}{c}\boldsymbol{\beta}_{\text {tangencial }} \\
(\boldsymbol{\%})\end{array}$ & $\begin{array}{c}\boldsymbol{\beta}_{\text {radial }} \\
(\boldsymbol{\%})\end{array}$ & $\begin{array}{c}\boldsymbol{\beta}_{\text {longitudinal }} \\
(\boldsymbol{\%})\end{array}$ & $\begin{array}{c}\boldsymbol{\beta}_{\text {volumétrica }} \\
(\boldsymbol{\%})\end{array}$ & $\mathbf{A}_{\mathbf{c}}$ \\
\hline Base & $4,280 \mathrm{a}$ & $1,954 \mathrm{a}$ & $0,502 \mathrm{a}$ & $6,600 \mathrm{a}$ & $2,208 \mathrm{a}$ \\
& $(13,54)$ & $(11,61)$ & $(19,18)$ & $(10,12)$ & $(9,43)$ \\
Meio & $4,024 \mathrm{a}$ & $1,714 \mathrm{a}$ & $0,238 \mathrm{~b}$ & $5,894 \mathrm{a}$ & $2,366 \mathrm{a}$ \\
& $(8,02)$ & $(14,31)$ & $(17,17)$ & $(8,30)$ & $(9,20)$ \\
Topo & $4,284 \mathrm{a}$ & $1,926 \mathrm{a}$ & $0,204 \mathrm{~b}$ & $6,314 \mathrm{a}$ & $2,252 \mathrm{a}$ \\
& $(15,10)$ & $(16,26)$ & $(11,81)$ & $(13,67)$ & $(15,56)$ \\
\hline
\end{tabular}

$\beta$ : Contração; $\mathrm{A}_{\mathrm{c}}$ : Fator anisotropico; Médias seguidas de mesma letra na mesma coluna são estatisticamente semelhantes pelo Teste de Tukey a 95\% de probabilidade; Valores entre parênteses referem-se ao coeficiente de variação.

A análise de variância indicou a inexistência de diferença estatística significativa para todas as contrações, exceto para a contração longitudinal (Tabela 2). Também não foi constatada diferença estatística significativa para o fator anisotrópico entre as diferentes posições longitudinais, indicando que a madeira apresenta estabilidade similar ao longo do fuste nesta espécie. Este resultado corrobora o fato da não segregação de toras quanto à altura em processos industriais para geração de produtos sólidos.

\section{Variação no sentido radial \\ Na figura 3 estão apresentados a variação das massas específicas básica e aparente da $T$. grandis} no sentido medula a casca.

Os valores médios apresentados na figura 3 indicam o aumento das massas específicas básica e aparente (12\%) no sentido medula-casca, sendo constatada diferença estatística significativa das posições intermediária e casca em relação aos valores obtidos próximo a medula. Este comportamento atende o primeiro modelo proposto por Panshin e de Zeeuw (1980), e é explicado provavelmente pela ocorrência de madeira juvenil, que está localizada próximo a medula da árvore, se estende desde a base até o topo e apresenta aumento progressivo das dimensões das células, bem como mudança das suas características e do arranjo celular. Normalmente possui fibras ou traqueóides mais curtos e parede celular mais fina, o que implica diretamente na massa específica. Para corroborar esta informação, vale ressaltar o trabalho realizado por Lima et al. (2009), que ao estudarem as dimensões anatômicas da madeira de $T$. grandis em função do espaçamento e da posição radial do tronco, constataram o aumento da espessura da parede celular, com valores de $4,47 \mu \mathrm{m}, 5,15 \mu \mathrm{m}$ e $5,62 \mu \mathrm{m}$, respectivamente para as posições medula, intermediária e casca. Além disso, é possível verificar a partir da figura 3, que entre as posições medula e porção intermediária ocorre um aumento acentuado na massa específica $\left(0,084\right.$ a $\left.0,090 \mathrm{~g} . \mathrm{cm}^{-3}\right)$, tendendo a estabilidade na porção intermediária à casca $\left(0,013\right.$ a $\left.0,014 \mathrm{~g} . \mathrm{cm}^{-3}\right)$, reforçando a hipótese de que as células estão se tornando estáveis e homogêneas.

Gonçalves et al. (2007) ao avaliarem a variação da massa específica de T. grandis procedentes de diferentes sítios e idades, obtiveram valores de 0,40 a $0,47 \mathrm{~g} . \mathrm{cm}^{-3}$ para as mostras de 5 anos procedentes do Espírito Santo, e 0,41 a 0,46 g. $\mathrm{cm}^{-3}$ para material de 12 anos procedentes de São Paulo. Tais valores são inferiores ao deste trabalho, e podem ser explicados principalmente pela diferença de idades. Melo et al. (2006) ao estudarem a madeira de T. grandis, proveniente de Areia, PB, determinaram em sua pesquisa, massa especifica entre 0,59 e $0,66 \mathrm{~g} . \mathrm{cm}^{-3}$, cujos valores podem ser considerados 
próximos aos determinados nesta pesquisa, e as diferenças podem ser atribuídas à idade, procedência e sitio de crescimento.

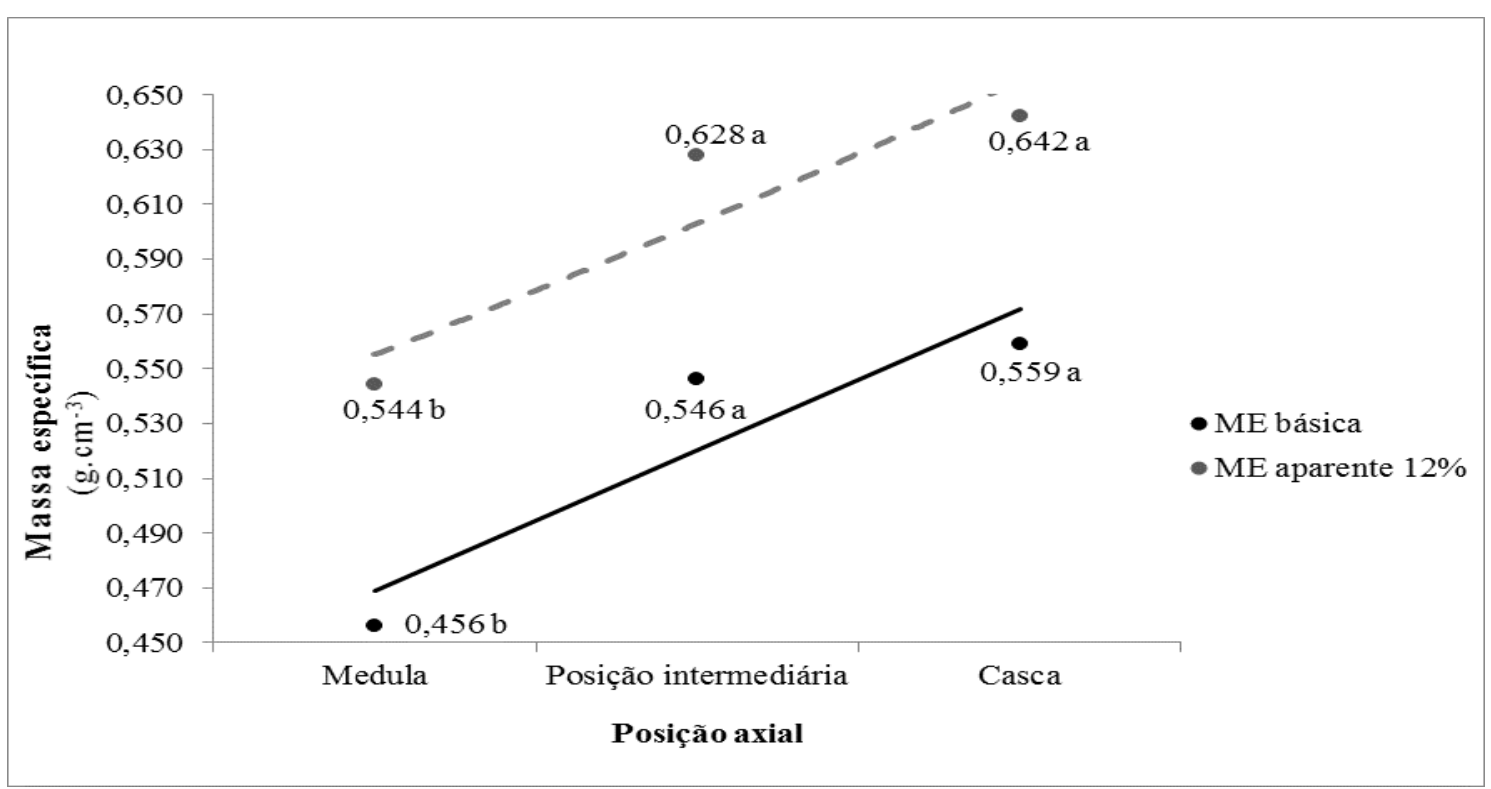

Figura 3. Variação das massas específicas básica e aparente a $12 \%$ no sentido radial de Tectona grandis. Figure 3. Variation of basic and apparent (12\%) density in the radial direction of Tectona grandis.

Na tabela 3 estão apresentados os valores médios das contrações tangencial, radial, longitudinal e volumétrica, e fator anisotrópico no sentido radial do tronco.

Tabela 3. Valores médios da retratibilidade da madeira no sentido radial do tronco de Tectona grandis. Table 3. Average values of shrinkage of wood in the radial direction of tree trunk of Tectona grandis.

\begin{tabular}{lccccc}
\hline Posição & $\boldsymbol{\beta}_{\text {tangencial }}$ & $\boldsymbol{\beta}_{\text {radial }}$ & $\boldsymbol{\beta}_{\text {longitudinal }}$ & $\boldsymbol{\beta}_{\text {volumétrica }}$ & $\mathbf{A}_{\mathbf{c}}$ \\
\hline \multirow{2}{*}{ Medula } & $4,799 \mathrm{a}$ & $2,064 \mathrm{a}$ & $0,484 \mathrm{a}$ & $7,172 \mathrm{a}$ & $2,388 \mathrm{a}$ \\
& $(14,35)$ & $(18,86)$ & $(12,82)$ & $(13,60)$ & $(13,55)$ \\
Intermediária & $3,708 \mathrm{~b}$ & $1,725 \mathrm{a}$ & $0,309 \mathrm{~b}$ & $5,661 \mathrm{~b}$ & $2,166 \mathrm{a}$ \\
& $(13,20)$ & $(14,78)$ & $(20,83)$ & $(11,94)$ & $(7,80)$ \\
Casca & $4,108 \mathrm{~b}$ & $1,819 \mathrm{a}$ & $0,246 \mathrm{~b}$ & $6,081 \mathrm{~b}$ & 2,280 \\
& $(12,64)$ & $(14,17)$ & $(18,40)$ & $(10,54)$ & $(12,61)$ \\
\hline
\end{tabular}

$\beta$ : Contração; $\mathrm{A}_{\mathrm{c}}$ : Fator anisotrópico; Médias seguidas de mesma letra na mesma coluna são estatisticamente iguais pelo Teste de Tukey a 95\% de probabilidade; valores entre parênteses referem-se ao coeficiente de variação.

Os resultados apresentados na tabela 3 indicam que a maioria das propriedades possuem diferenças significativas em nível de $95 \%$ de probabilidade, entre as posições radiais medula e casca, exceto a contração radial e a anisotropia de contração. Observa-se que em nenhuma das propriedades estudadas existe diferença significativa entre a posição intermediária e a casca. Esse fato está associado à variação de massa específica, a qual apresentou o mesmo comportamento estatístico (Figura 3), que possivelmente foi influenciada pela presença de madeira juvenil na região próxima à medula, e uma tendência de estabilização das células na região próximo a casca, com formação de madeira adulta. Em complemento a este fato, vale ressaltar que a madeira juvenil por possuir maior ângulo microfibrilar na camada $S_{2}$ da parede celular, parede celular mais fina e menor percentual de lenho tardio, normalmente afeta negativamente as propriedades de contração e anisotropia, gerando propriedades físicas mais instáveis (PANSHIN; DE ZEEUW, 1980).

Em comparação com a literatura, Finger et al. (2003) ao avaliarem a qualidade da madeira de $T$. grandis oriunda do primeiro desbaste, obtiveram valores no ensaio de retratibilidade que indicaram que a madeira próxima à medula é mais estável $(\mathrm{Ac:} 1,72)$ quando comparada com a madeira próximo ao 
alburno (Ac: 2,00), sendo estes resultados contrários aos determinados no presente trabalho, mas que, de certa forma, poderiam ser explicados pela composição química e pela massa específica das diferentes regiões. Verificou-se ainda que, Silva et al. (2006) ao estudarem as propriedades de E. grandis de 4 diferentes idades, observaram aumento crescente das contrações no sentido medula a casca, e fator anisotrópico praticamente constante nas diferentes posições avaliadas. Teixeira (2008) também obteve tendência de aumento das contrações no sentido medula a casca, porém para o fator anisotrópico a autora não encontrou tendência definida.

Com base nos resultados apresentados na tabela 3, pela pequena diferença entre os valores nas diferentes posições radiais, mesma classificação quanto ao fator anisotrópico, bem como pela inexistência de diferença estatística significativa, é possível reforçar a idéia da não segregação de peças em diferentes classes de qualidade durante o processamento e utilização da madeira.

\section{CONCLUSÕES}

Com base nos resultados obtêm-se as seguintes conclusões:

- Existe uma tendência de redução da massa específica da base ao meio, com ligeiro aumento do meio ao topo do tronco;

- Tanto a contração tangencial como a massa específica aparente são maiores próximos a casca e menores próximos a medula;

- Pode se constatar que existe um decréscimo da contração tangencial nas regiões próximas do topo da árvore e que na base a contração aumenta com o aumento da massa específica.

- Não há necessidade de segregação de peças em classes de qualidade tanto no sentido axial quanto no sentido radial.

- Os resultados demonstram que as árvores de T. grandis utilizadas neste estudo possuem boa estabilidade dimensional, resultando desta forma, em madeira com qualidade média frente à sua utilização em produtos sólidos.

\section{REFERÊNCIAS}

ASSOCIAÇÃO BRASILEIRA DE PRODUTORES DE FLORESTAS PLANTADAS (ABRAF). Anuário estatístico da ABRAF 2013, ano base 2012. Brasília, 2013, 148 p.

ARRUDA, T. P. M.; LOGSDON, N. B.; SILVA, J. R. M.; LIMA, D. F.; PROTASIO, T. P. Variação das densidades aparente e básica da madeira de Tectona grandis de $\mathbf{1}^{\circ} \mathbf{d e s b a s t e . ~ V i t o ́ r i a : ~ U F E S , ~ 2 0 1 2 , ~} 10$ p.

BAILLÈRES, H.; DURAND, P. Y. Non-destructive techniques for wood quality assessment of plantation grown teak. Bois et Forest dês Tropiques, Montpellier, v. 54, n. 263, p. 17 - 20, 2000.

CASTRO, F. Y.; RAIGOSA, J. Crescimiento y Propriedades Fisico-Mecanicas de La madera de teca (Tectona grandis) de 17 años de edad em San Joaquin de Abangares, Costa Rica, 2000. Disponível em: 〈http://www.mag.go.cr/rev_agr/v.24n.2_007.pdf >.Acesso em: 09/04/2013.

COMISSÃO PANAMERICANA DE NORMAS TÉCNICAS. COPANT: métodos de ensayos físicos y mecânicos. [S.1], 1972.

DURLO, M. A.; MARCHIORI, J. N. Tecnologia da madeira. Santa Maria: CEPEF/FATEC, 1992, 33 p.

FINGER, A. F.; FINGER, Z.; LONSDON, N. B. Qualificação da madeira de teca, Tectona grandis 1. f., oriunda do primeiro desbaste comercial, com vistas a seu aproveitamento. Madeira: Arquitetura e Engenharia, São Carlos, v. 4, n. 10, p. 1 - 31, 2003.

FLÓREZ, J. B. Caracterização tecnológica da madeira jovem de teca (Tectona grandis L. f). 85 f. Dissertação (Mestrado em Ciência e Tecnologia da Madeira) - Universidade Federal de Lavras, Lavras, 2012.

FOREST PRODUCTS LABORATORY (FPL). Wood handbook: wood as an engineering material. Madison: U.S. Department of Agriculture, Forest Service, 2010, 508 p. 
GONÇALVES, M. P. M.; COFFLER, R.; CARVALHO, A. M.; GARCIA, R. A. Variação radial da densidade básica e do comprimento das fibras da madeira de Tectona grandis L. Floresta e Ambiente, Seropédica, v. 14, n. 1. p. 70 - 75, 2007.

GUTIÉRREZ, R. C.; ROMERO, E. J.; CUNUHAY, P. S.; BLANCO, G. L.; FONSECA, C. S. Analisis comparativo de las propiedades físico-mecánicas de la madera de teca (Tectona grandis L.F) de Quevedo y Balzar. Ciência y Tecnologia, Bio-Bio, v. 1, n. 2, p. 55 - 63, 2008.

KOLlMANN, F. F. P.; CÔTÉ, W. A. Principles of wood science and technology: I Solid Wood. New York: Springer, 1968, v. 1, 592 p.

LIMA, I. L.; FLORSHEIM, M. B.; LONGUI, E. L. Influência no espaçamento em algumas propriedades físicas da madeira de Tectona grandis Linn. Cerne, Lavras, v. 15, n. 2, p. 244 - 250, 2009.

LOPES, C. S. D.; NOLASCO, A. M.;TOMAZELLO FILHO, M.; DIAS, C. T. S.; PANSINI, A. Estudo da massa específica básica e da variação dimensional de três espécies de eucalipto para a indústria moveleira. Ciência Florestal, Santa Maria, v. 21, n. 2, p. 315 - 322, 2011.

MELO, R. R.; PAES, J. B.; LIMA, C. R.; FERREIRA, A. G. Estudo da variação radial da densidade básica de sete madeiras do semi-árido. Revista Científica Eletrônica de Engenharia Florestal, Garça, v. 5, p. 1 - 8, 2006.

MELO, J. E.; CORADIN, V. T. R.; MENDES, J. C. Classes de densidade para madeiras da Amazônia Brasileira. In: $6^{\circ}$ CONGRESSO FLORESTAL BRASILEIRO, FLORESTAS E MEIO AMBIENTE: CONSERVAÇÃO E PRODUÇÃO, PATRIMÔNIO SOCIAL. Anais... Campos do Jordão, SP, 1990, p. $695-699$.

MIRANDA, I.; SOUSA, V.; PEREIRA, H. Wood properties of teak (Tectona grandis) from a mature unmanaged stand in East Timor. Journal of Wood Science, Hokkaido, v. 57, n. 3, p. 171 - 178, 2011.

MOTTA, J. P. Propriedades tecnológicas da madeira de Tectona grandis L. f. proveniente do Vale do Rio Doce, Minas Gerais. 112 f. Dissertação (Mestrado em Ciências Florestais) - Universidade Federal do Espírito Santo, Jerônimo Monteiro, 2011.

PANDEY, D.; BROWN, C. La teak: una visión global. Revista Internacional de Silvicultura e Industrias Forestales, Rome, v. 51, n. 2, p. 3 - 13, 2000.

PANSHIN, A. J.; ZEEUW, C. Text book of wood technology. 4. ed. New York: McGraw Hill.

ROQUE, R. M.; LEDEZMA, V. A. Efecto del espaciamiento en plantación sobre dos propiedades físicas de madera de teca a lo largo del fuste. Madera y Bosques, México, v. 9, n. 2, p. 15 - 27, 2003.

SILVA, J. C.; OLIVEIRA, J. T. S.; XAVIER, B. A.; REZENDE, V. C. Variação da retratibilidade da madeira de Eucalyptus grandis ex. Maiden, em função da idade e da posição radial. Árvore, Viçosa, v. 30, n. 5, p. 803 - 810, 2006.

TEIXEIRA, B. M. R. Variabilidade radial e longitudinal de propiedades da madeira de angicovermelho (Anadenanthera peregrina (L.) Speg.). 52 f. Dissertação (Mestrado em Ciências Florestais) Universidade Federal de Viçosa, Viçosa, 2008.

TSOUMIS, G. T. Science and technology of Wood: Structure, properties, utilization. New York: Chapmann e Hall, 1991, 479 p. 
FLORESTA, Curitiba, PR, v. 45, n. 4, p. 671 - 680, out. / dez. 2015. Bonduelle, G. M. et al. 